\title{
Development of Virtual Illumination Functions for Thermographic NDT
}

\author{
by P. Venegas*`*, J. Perán**, R. Usamentiaga***, I. Sáez de Ocáriz* \\ * Aeronautical Technologies Centre, Juan de la Cierva 1, 01510 Miñano, Spain, pablo.venegas@ctaero.com, \\ idurre.saezdeocariz@ctaero.com \\ ** National Distance Education University, Department of Applied Mathematics, C/ Juan del Rosal 12, 28040 \\ Madrid, Spain, jperan@ind.uned.es \\ *** University of Oviedo, Department of Computer Engineering, C/ San Francisco 1, 33003 Oviedo, Spain, \\ rusamentiaga@uniovi.es
}

\begin{abstract}
This study develops and analyses a novel mathematical approach for thermographic NDT data processing. This approach is based on the definition of a simplified 2D thermal diffusion model to which usual processing algorithms are applied to produce analysis functions, which considers information related to temperature values, material properties and heating conditions. The analysis of the developed functions has been conducted with thermal data computationally generated in order to provide broad variety of case studies regarding different types of defects as well as stimulations. The results obtained have demonstrated that these functions may provide information useful in defect characterisation.
\end{abstract}

\section{Introduction}

Infrared thermography (IRT) is a versatile technology currently used in numerous applications $[1,2,3]$. The special characteristics of this technology together with technological advances and costs reduction have made IRT spread quickly in the recent years. IR sensors are becoming more and more sensitive and robust with higher spatial and thermal resolution and higher acquisition frame rates. Processing algorithms play an important role in the improvement of the measures provided by IRT and are also a key point in the continuous technological developments.

The application of IRT as a non-destructive testing (NDT) tool is increasing quickly. IRT is capable of inspecting materials rapidly, at distance and without contact for integrity evaluation without producing damage or affecting their properties. Some of the most common data processing techniques used in IRT NDT are Thermographic Signal Reconstruction [4], which is based on a polynomial fitting mathematical process, spectral analysis [5], which is based on the Fourier Transform mathematical process, and Principal Component Analysis [6], which is based on the Singular Value Decomposition mathematical process, among others.

This study develops a novel mathematical approach for IRT NDT data processing that is based on a 2D thermal diffusion model obtained by projecting the general 3D thermal diffusion equation onto a coordinate plane [7]. The subsequent application of usual mathematical algorithms to this simplified model leads to the Virtual Illumination Functions (VIF), which provide additional information about the detected defects. This study analyses the properties of these VIFs as well as the specific graphical representation of defects and the improvement provided in the detection level.

\section{Virtual illumination functions}

\subsection{D simplified diffusion model}

The proposed Virtual Illumination Functions are developed from an anisotropic 2D thermal diffusion model that is generated by projecting the features of a 3D isotropic thermal diffusion model onto a coordinate plane. The 3D thermal diffusion differential equation may accurately model the behaviour of an IRT NDT inspection under certain simplifying hypotheses. Assuming that the material under inspections is opaque, heterogeneous and isotropic, no chemical reaction occurs as a consequence of the heat irradiation, no internal heat sources exist inside the material, the thermophysical properties of the medium remain constant during the inspection and are independent of temperature, the external surface behaves as a grey body and the incident energy is diffuse and homogeneously distributed along the surface, the thermal process that takes place in IRT NDT inspections may be properly represented by the heat diffusion equation (1),

$$
\begin{aligned}
& \frac{\partial u(x, t)}{\partial t}-\nabla \cdot(c(x) \nabla u(x, t))=F(x, t) \\
& x \in \mathfrak{R}^{3}, t \geq 0, c(x) \in \mathfrak{R}^{+}
\end{aligned}
$$



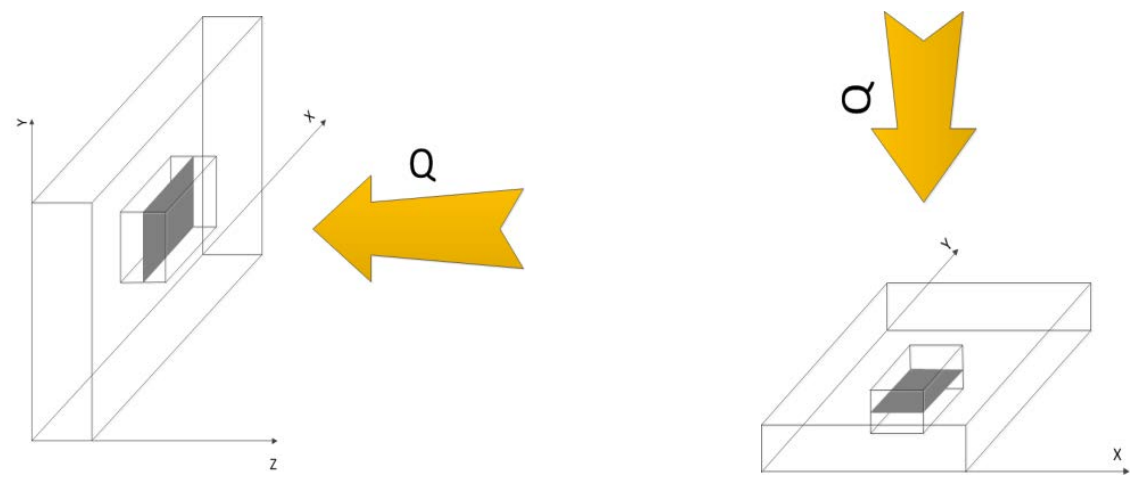

Fig. 1. 3D thermal diffusion model (left) and 2D simplified thermal diffusion model (right).

where $u$ is the temperature at each material point, $c$ is the thermal diffusivity of the material $\left(c=k /\left(\rho c_{p}\right)\right), k$ is the thermal conductivity, $\rho$ is the material density, $c_{p}$ is the specific heat at constant pressure and $\nabla$ is the nabla operator with respect to the spatial variables, and $F$ is the heat sources. as follows:

The result of the projection procedure (figure 1) is a differential equation whose expanded form is represented

$$
\begin{aligned}
& \frac{\partial u(x, t)}{\partial t}-\nabla \cdot(C(x, t) \nabla u(x, t))=F(x, t) \\
& x \in \mathfrak{R}^{2}, t \geq 0
\end{aligned}
$$

where $C(x, t)$ is the projected thermal diffusivity matrix, which consists in a $2 \times 2$ positive definite matrix with a general form:

$$
\left(\begin{array}{ll}
c_{11}(x, t) & c_{12}(x, t) \\
c_{21}(x, t) & c_{22}(x, t)
\end{array}\right)
$$

The subsequent application of usual processing algorithms to this simplified anisotropic model, e.g. time derivatives or spectral analysis, and the appropriate operations conduct to a series of analysis functions. These functions, here defined as Virtual Illumination Functions, combine information about temperature evolution and material properties with information related to the heating source characteristics.

\subsection{Virtual illumination functions for successive derivation}

Different common processing algorithms may be applied to the simplified anisotropic model to produce Virtual Illumination Functions, including time derivatives, spectral analysis and principal component analysis. The general procedure to produce VIFs consists in the application of a specific processing algorithm to the simplified 2D diffusion equation and subsequently operating the result to reach a form analogous to the initial simplified diffusion equation. The term obtained analogous to the initial heating source is the VIF corresponding to the considered processing algorithm.

The most straightforward VIFs are obtained by applying successive derivatives to the equation (2). Thus, the result after first derivation may be expressed as:

$$
\begin{aligned}
& \frac{\partial^{2} u(x, t)}{\partial t^{2}}-\frac{\partial}{\partial t}(\nabla \cdot(C(x, t) \nabla u(x, t)))=\frac{\partial F(x, t)}{\partial t} \\
& \frac{\partial^{2} u(x, t)}{\partial t^{2}}-\nabla \cdot\left(\frac{\partial C(x, t)}{\partial t} \nabla u(x, t)\right)-\nabla \cdot\left(C(x, t) \nabla \frac{\partial u(x, t)}{\partial t}\right)=\frac{\partial F(x, t)}{\partial t}
\end{aligned}
$$

We now apply the change of variable $v=\partial u / \partial t$ to obtain:

$$
\frac{\partial v(x, t)}{\partial t}-\nabla \cdot\left(\frac{\partial C(x, t)}{\partial t} \nabla u(x, t)\right)-\nabla \cdot(C(x, t) \nabla v(x, t))=\frac{\partial F(x, t)}{\partial t}
$$


Finally, reorganising terms looking for the form of the initial equation we reach the following expression:

$$
\frac{\partial v(x, t)}{\partial t}-\nabla \cdot(C(x, t) \nabla v(x, t))=\frac{\partial F(x, t)}{\partial t}+\nabla \cdot\left(\frac{\partial C(x, t)}{\partial t} \nabla u(x, t)\right)
$$

We can recognise in the equation (4) the form of the initial simplified 2D diffusion equation after applying first derivation and a change of variable. Looking at the term in the left, we have now a sum of terms that include information about the stimulation conditions $(\partial F / \partial t)$, the material properties $(\partial C / \partial t)$ and the thermal evolution of the material $(\nabla u)$. This sum of terms is the so-called Virtual Illumination Function for the first derivative and is represented as:

$$
G_{D E R_{1}}(x, t)=\frac{\partial F(x, t)}{\partial t}+\nabla \cdot\left(\frac{\partial C(x, t)}{\partial t} \nabla u(x, t)\right)
$$

Successive derivation may be applied to the equation (2) in order to obtain VIFs for successive derivatives. The procedure is the same as for the first derivative so a change of variable and a reorganization of terms enable to reach the desired functions. The VIFs for derivatives from 2 to 4 are shown below:

$$
\begin{aligned}
G_{D E R_{2}}(x, t)= & \frac{\partial^{2} F(x, t)}{\partial t^{2}}+\nabla \cdot\left(\frac{\partial^{2} C(x, t)}{\partial t^{2}} \nabla u(x, t)\right)+2 \nabla \cdot\left(\frac{\partial C(x, t)}{\partial t} \nabla v(x, t)\right) \\
G_{D E R_{3}}(x, t)= & \frac{\partial^{3} F(x, t)}{\partial t^{3}}+\nabla \cdot\left(\frac{\partial^{3} C(x, t)}{\partial t^{3}} \nabla u(x, t)\right)+3 \nabla \cdot\left(\frac{\partial^{2} C(x, t)}{\partial t^{2}} \nabla v(x, t)\right) \\
& +3 \nabla \cdot\left(\frac{\partial C(x, t)}{\partial t} \nabla w(x, t)\right) \\
G_{D E R_{4}}(x, t)= & \frac{\partial^{4} F(x, t)}{\partial t^{4}}+\nabla \cdot\left(\frac{\partial^{4} C(x, t)}{\partial t^{4}} \nabla u(x, t)\right)+4 \nabla \cdot\left(\frac{\partial^{3} C(x, t)}{\partial t^{3}} \nabla v(x, t)\right) \\
& +6 \nabla \cdot\left(\frac{\partial^{2} C(x, t)}{\partial t^{2}} \nabla w(x, t)\right)+4 \nabla \cdot\left(\frac{\partial C(x, t)}{\partial t} \nabla s(x, t)\right)
\end{aligned}
$$

where $v=\partial u / \partial t, w=\partial v / \partial t=\partial^{2} u / \partial t^{2}$ and $s=\partial w / \partial t=\partial^{3} u / \partial t^{3}$.

Other VIFs may be produced by the application of different processing techniques, such as Fourier Transformation, Principal Component Analysis and Higher Order Statistics, and a similar deductive procedure based on changes of variable and terms reorganization looking for the form of the equation (2).

\section{Analysis of Virtual Illumination Functions}

\subsection{Thermographic data used in the study}

The data necessary to calculate the VIFs are the temperature-time history $u(x, t)$, the projected diffusivity-time history $C(x, t)$ and the stimulation-time history $F(x, t)$. The temperature values $u(x, t)$ may be obtained directly by the IR sensor in a real inspection. However, in this study, thermographic data computationally generated was used in order to avoid the influence due to the imperfections of real situations in the behaviour of the VIFs. The lack of issues related to the noise produced by the IR sensor, the stimulation heterogeneities in the surface of the samples and the uncertainties regarding the characteristics of the base material and defects enable to determine the fundamental features and characterization capacities of the VIFs.

The temperature time-history was generated by the equation (1), considering parameters that represented IRT NDT tests and the inspected materials, and programming an algorithm for numerical resolution. Different stimulation conditions were considered, all of them modelling the injection of energy in the external surface of the inspected sample. Stimulation conditions produced by step heating, pulsed heating and step cooling were modelled. The amount of energy involved in all the stimulation situations was $10 \mathrm{KJ}$. The step stimulation produced an inner heat flow in the case of heating and an outer heat flow in the case of the cooling. In both cases the stimulation was applied during 10 seconds. The pulsed stimulation consisted in an initial injection of the energy producing an instantaneous temperature increase in the external surface.

The type of base material modelled in this study was standard carbon fibre reinforced polymer (CFRP) due to the great interest and wide use in the aeronautical industry. This material was modelled with diffusivity values taken from 


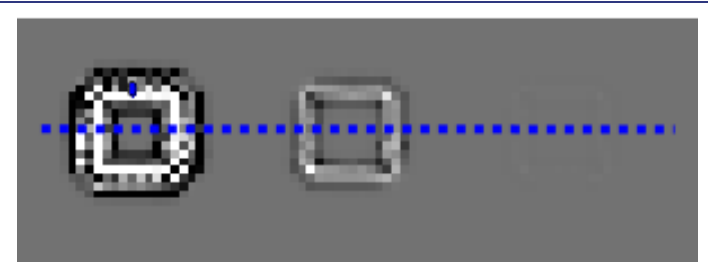

(a)

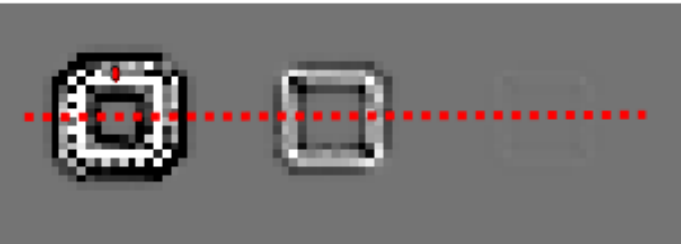

(b)

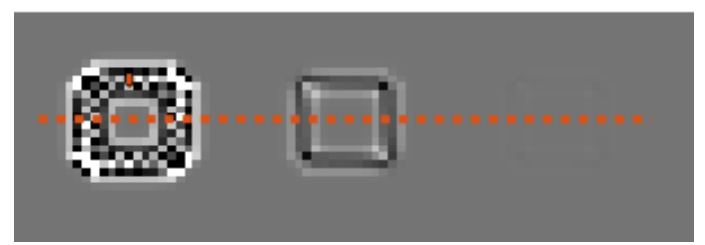

(c)

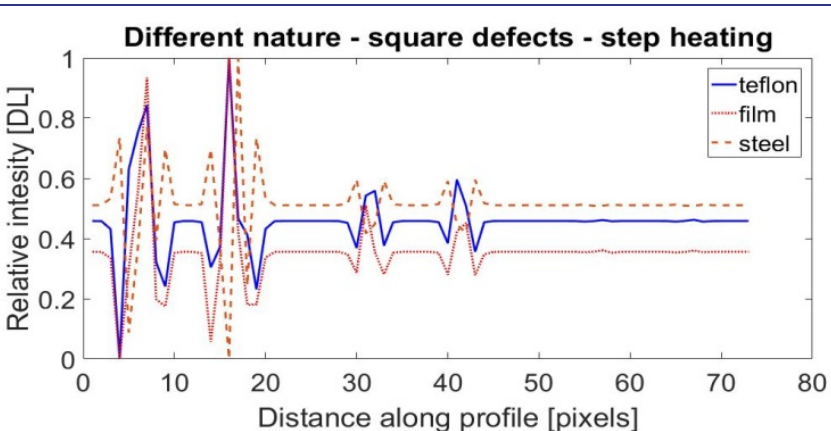

(d)

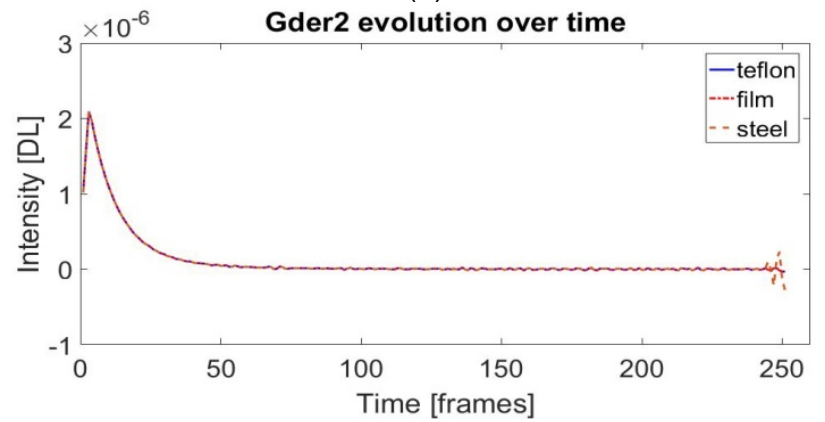

(e)

Fig. 2. Results obtained in the analysis of the dependency of Gder2 on defect nature. Figures (a), (b) and (c) are results obtained at the same instant for Teflon, film and steel, respectively. Figures (d) and (e) show the spatial response and time history of the corresponding profiles and points in figures (a), (b) and (c).

bibliographic sources so the values used for the different directions were $\alpha_{x}=7.0 \pm 2 \cdot 10^{-7} \mathrm{~m}^{2} / \mathrm{s}, \alpha_{y}=3.5 \pm 2 \cdot 10^{-7} \mathrm{~m}^{2} / \mathrm{s}$ and $\alpha_{z}=1.0 \pm 0.3 \cdot 10^{-6} \mathrm{~m}^{2} / \mathrm{s}$. Different types of defects were modelled in the CFRP material to provide a wide array of results. Defects that simulated different types of materials, such as small pieces of Polytetrafluoroethylene (PTFE), protective films and thin metal sheets, were programmed. Moreover several depths and different shapes were considered for defect characterization.

The stimulation-time history $F(x, t)$ was also computationally generated according to the values used in the generation of the temperature-time history, and the projected diffusivity values $C(x, t)$ were obtained by means of a projected thermal diffusivity analysis [7].

\subsection{Results obtained in the analysis}

\subsubsection{Dependency on the nature of defects}

The behaviour of the VIFs with respect to the nature of defects has been analysed. Materials simulating common defects in CFRP have been modelled each one with different thermal properties. The defect shape, size and depth, as well as the type of stimulation, were fixed in this analysis only varying the nature of the defects in order to avoid unwanted influence in the results.

Some representative results about the dependency on the nature of defects of the virtual functions for second derivative are shown in figure 2. Three square defects located at different depths, deeper to the right, were modelled in each test (figure $2 \mathrm{a}, 2 \mathrm{~b}$ and $2 \mathrm{c}$ ). Spatial profiles crossing the defects at the same instant are visualized in figure $2 \mathrm{~d}$ and the evolution over time of the same defective point corresponding to different defects is shown in figure $2 \mathrm{e}$.

It can be observed from these results that the nature of the defects produces a different response in the virtual illumination functions. Those defects with thermal diffusivities greater than the base material generate negative gradients in the edges of the defects, while those defects with diffusivities smaller than the base material generate positive gradients. The results provided by PTFE and film defects in the spatial profile are contrary to those provided by the steel sheets. This special response enables to identify the nature of defects and characterize it.

The signal level of virtual functions varies with the nature of the defects. The greater the difference of thermal diffusivities between the base material and the defect, the greater the signal level. The sign of the ratio of thermal diffusivities has no influence on the sign of the resulting signal. The signal will be higher with higher difference in diffusivities regardless of whether it is positive or negative.

It is observed that the signal increase in the edges of the defects with respect to the signal in the healthy zones is constant in absolute value for the same type of stimulation, independently of the nature of the defects, so it enables to characterize the size of the defects accurately.

The signal of the VIF for second derivative varies over time with a high slope at the initial instants. The signal reaches $90 \%$ of its final value quickly and has a horizontal asymptote at zero value. According to figure $2 \mathrm{e}$, the nature of the defects hardly influences the temporal evolution of the virtual illumination signal for a given stimulation. 


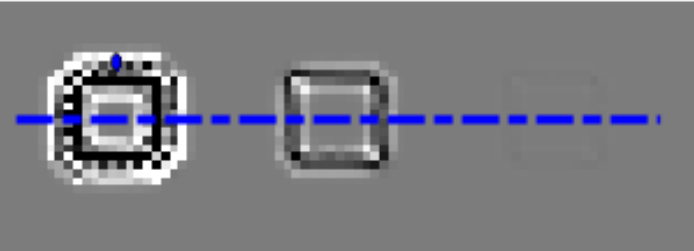

(a)

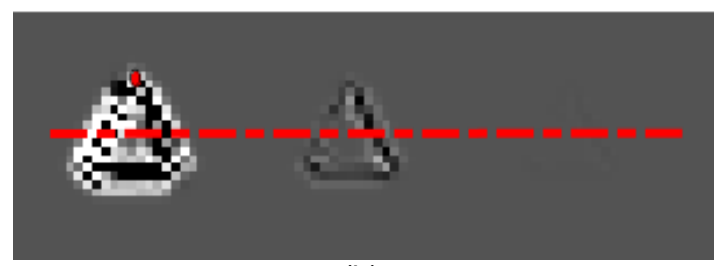

(b)

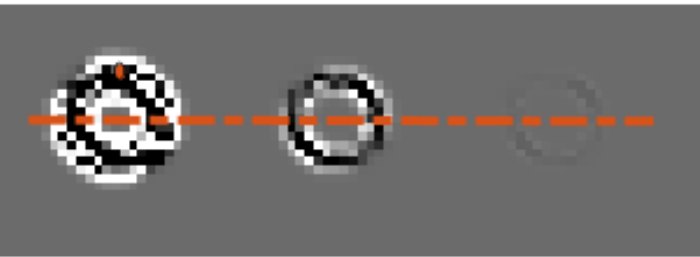

(c)

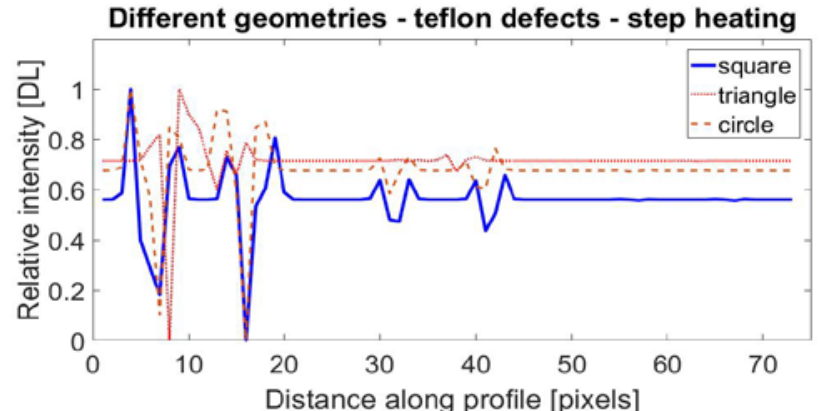

(d)

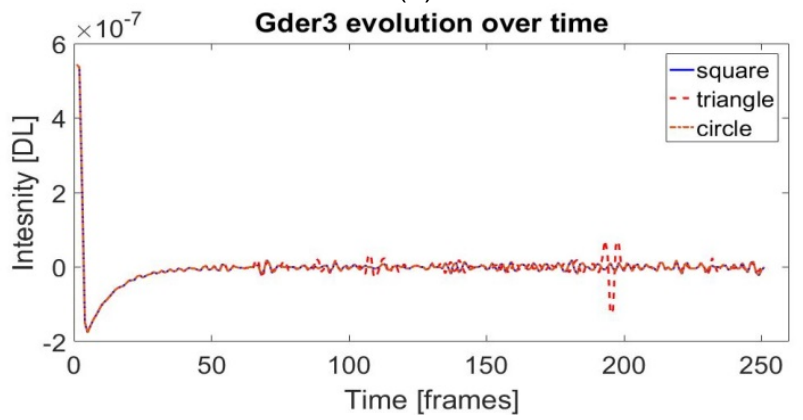

(e)

Fig. 3. Results obtained in the analysis of the dependency of Gder3 on defect shape. Figures (a), (b) and (c) are results obtained at the same instant for Teflon, film and steel, respectively. Figures (d) and (e) show the spatial response and time history of the corresponding profiles and points in figures (a), (b) and (c).

\subsubsection{Dependency on the shape of defects}

The behaviour of the VIFs with respect to the shape of defects has been analysed. Different shapes have been modelled to study the capacity of identifying the edges of defects and reproduce their geometry. The defect nature and depth, as well as the type of stimulation, were fixed in this analysis only varying the shape of the defects in order to avoid unwanted influence in the results.

Some representative results about the dependency on the shape of defects of the virtual functions for third derivative are shown in figure 3. Three defects located at different depths, deeper to the right, with square, triangular and circular shapes were modelled in each test (figure $3 a, 3 b$ and $3 c$ ). The spatial profiles crossing the defects at the same instant are visualized in figure $3 \mathrm{~d}$ and the evolution over time of defective points with similar behaviour is shown in figure 3e.

It can be observed from these results that the contour of the defects shows signal values different from the rest of the defective points and from the healthy material. The complete geometry of the defect could be reconstructed by identifying the maximum and minimum signal points in the spatial profiles. Furthermore, the area affected by the heat flow disturbed by the existence of a defect is represented as an area of evolution with abrupt slope. Those zones that do not have lateral heat gradients during the stimulation take the same signal value, including the central areas of the defects. The deepest defects show a lower signal at the edges of the defect becoming imperceptible for very deep ones.

As in the previous case, the signal of the VIF for third derivative and step heating stimulation varies over time with a high slope at the initial instants. The signal reaches $90 \%$ of its final value quickly and has a horizontal asymptote at zero value. According to figure $3 e$, the shape of the defects hardly influences the temporal evolution of the signal of the virtual illumination functions for a given stimulation.

\subsubsection{Dependency on the type of stimulation}

The behaviour of the VIFs with respect to the type of stimulation has been analysed. Different stimulations have been modelled to study the influence on characterizing defects under different test conditions. Step heating and pulsed heating have been considered because of the extended use in IRT NDT. Step cooling has also been considered in the analysis in order to cover a wider field of applications including frigorific cycles. The defect shape, nature and size were fixed in this analysis only varying the type of stimulation to avoid unwanted influence in the results.

Some representative results about the dependency on the type of stimulation of the virtual functions for first derivative are shown in figure 4 . Three square defects located at different depths, deeper to the right, were modelled in each test (figure $4 \mathrm{a}, 4 \mathrm{~b}$ and $4 \mathrm{c}$ ). The spatial profiles crossing the defects at the same instant are visualized in figure $4 \mathrm{~d}$ and the evolution over time of the same defective point is shown in figure $4 \mathrm{e}$. 


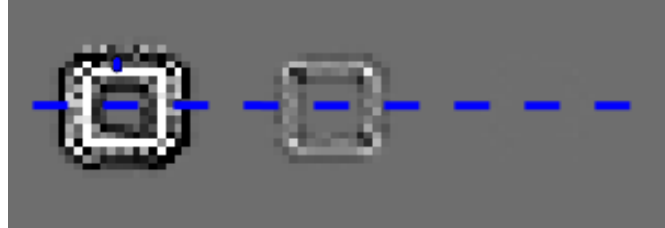

(a)

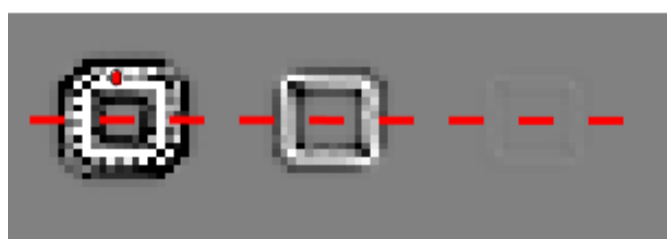

(b)

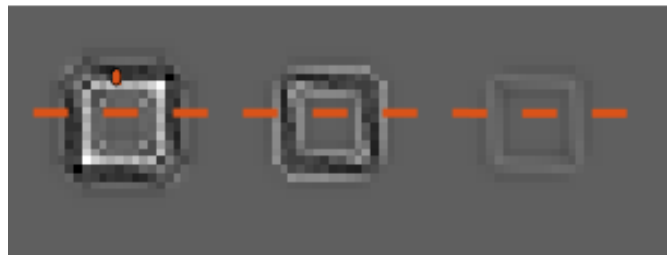

(c)

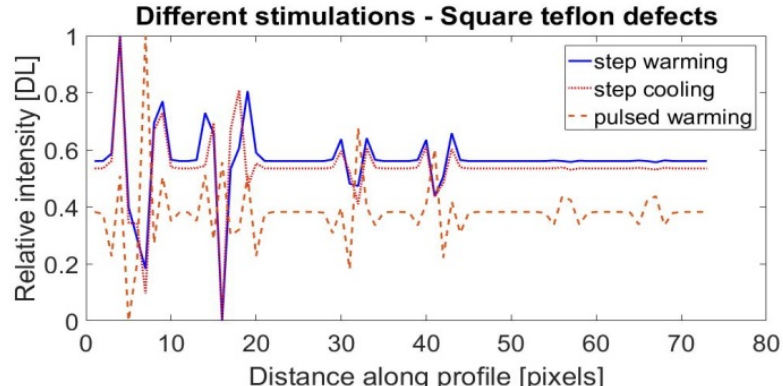

(d)

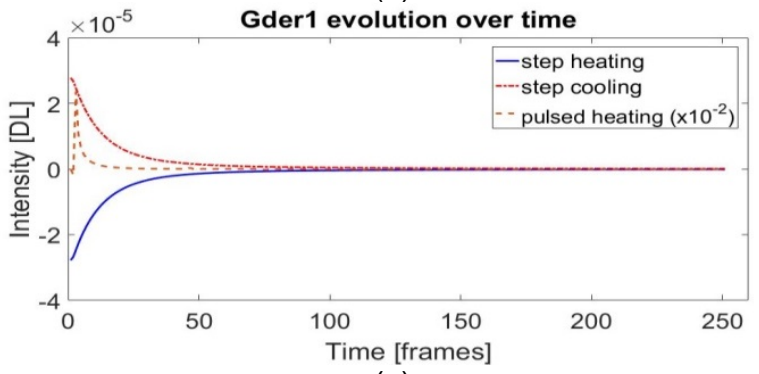

(e)

Fig. 4. Results obtained in the analysis of the dependency of Gder1 on the stimulation. Figures (a), (b) and (c) are results obtained at the same instant for Teflon, film and steel, respectively. Figures (d) and (e) show the spatial response and time history of the corresponding profiles and points in figures (a), (b) and (c).

It can be observed from these results that the step heating and step cooling stimulations provide almost the same spatial profile pattern, while the pulsed stimulation, for the same applied energy, provides greater signal and identification of deeper defects. The edges of the defects show signal values different from the rest of the defective points and from the healthy material for all the stimulations. Those zones that do not have lateral heat gradients take the same signal value, including the central areas of the defects.

As in the previous cases, the signal of the VIF for the first derivative and step heating stimulation varies over time with a high slope at the initial instants, reaches $90 \%$ of its final value quickly and has a horizontal asymptote at zero value. According to figure $4 \mathrm{e}$, the step cooling stimulation shows a very similar tendency but with an opposite sign of the slope compared to the heating case. The signal for pulsed stimulation has a behavior similar to the case of cooling stimulation, where the signal experiences a rapid pulse increase and then descends very quickly until reaching an asymptotic value at zero.

\subsubsection{Quantification of the detection level}

Two metrics have been used to quantify the detection of defects provided by the VIFs: the SNR and the sharpness. The SNR metric is usually used in IRT NDT to quantify the severity of a defect by comparing its signal to the signal of the sane material. This metric requires the preliminary location of two measurement areas, one for the defect and another for the undamaged reference. The defective area encloses pixels of the image that correspond to defects. The reference area encloses non-defective pixels, located close to the defective area to reduce the effect of the stimulation conditions. Different expressions may be applied to measure the SNR. The SNR is calculated in this study by the expression (9), where $D e f_{\mu}$ is the arithmetic mean of the intensity of pixels inside the defective area, $R e f_{\mu}$ is the arithmetic mean of the intensity of pixels inside the reference area, and $\operatorname{Ref}_{\sigma}$ is the standard deviation of the intensity of pixels inside the reference area.

$$
S N R=20 \log _{10}\left(\frac{\left|D e f_{\mu}-\operatorname{Re} f_{\mu}\right|}{\operatorname{Re} f_{\sigma}}\right)
$$

The sharpness is a magnitude for image quality assessment that provides information about the contrast of the objects within the scene. Different expressions may be applied to measure the sharpness. The sharpness is calculated in this study by the equation (10), which is a general gradient definition that may be used to evaluate the sharpness, where $f$ is the intensity values distribution in a given image, $x$ and $y$ are the pixel position for sharpness calculation and $M$ and $N$ are the number of horizontal and vertical pixels of the measuring area.

$$
S H A=\sum_{x=0}^{M-2} \sum_{y=0}^{N-1}(f(x+1, y)-f(x, y))^{2}+\sum_{x=0}^{M-1} \sum_{y=0}^{N-2}(f(x, y+1)-f(x, y))^{2}
$$




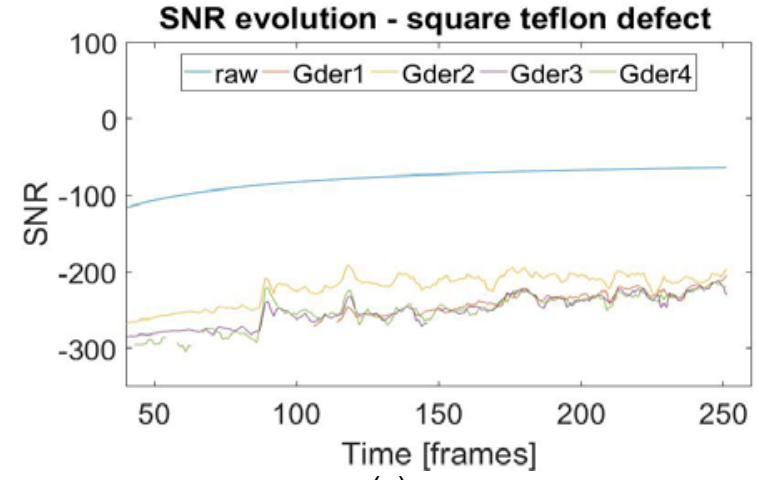

(a)

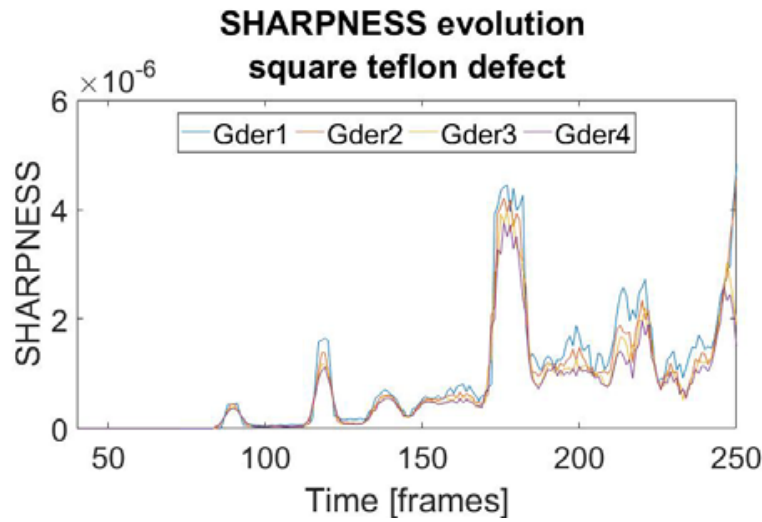

(b)

Fig. 5. Results of SNR and sharpness produced by $G_{d e r 1}, G_{d e r 2}, G_{d e r 3}$ and $G_{d e r 4}$ at the same conditions of stimulation and defect characteristics.

The quantification metrics were applied to the generated thermographic data and the results obtained were analysed. Some representative results for SNR and sharpness are shown in figure 5. Each metric is calculated for a defect with specific nature, shape and depth subject to a specific type of stimulation.

The SNR in figure 5 a shows that the values provided by the VIFs for all the derivatives have a similar behaviour with relative extrema located at the same instants. The highest SNR signal value was provided by the second derivative. However, the SNR values for all the VIFs were lower than the value produced by the raw thermographic data. This issue makes the VIFs theoretically unsuitable for improvement of detection level.

The sharpness metric also shows the similar tendencies provided by all the VIFs. However, unlike the SNR metric, the sharpness produces a better quality signal showing clearly the instants for relative maxima and the absolute maximum. It can be observed in figure $5 b$ that the initial instants of sharpness produce null value until the first relative maximum is reached. This stage with null sharpness value corresponds to the time during which the specific defect remains undetected. This issue demonstrates the capacity of the VIFs to characterize the depth of defects.

\section{Conclusions and future work}

A novel mathematical approach for thermographic NDT data processing is developed and analysed in this study. This approach is based on the definition of a simplified 2D thermal diffusion model to which usual processing algorithms are applied to produce analysis functions, the so-called Virtual Illumination Functions, which consider information related to temperature values, material properties and heating conditions.

The properties of the VIFs corresponding to successive derivatives as well as the improvement provided in the defect detection level have been analysed. This analysis has been conducted with thermal data computationally generated in order to provide broad variety of case studies regarding different types of defects as well as stimulations avoiding the influence in the results of the imperfections of real cases.

Stimulation conditions produced by step heating, pulsed heating and step cooling were modelled, all of them considering the injection of energy applied to the external surface of the inspected sample. Standard carbon fibre reinforced polymer (CFRP) was the type of base material modelled, including defects of different shape, nature and depth in order to provide a wide range of results.

The results obtained have demonstrated that the VIFs may provide information useful in defect characterisation tasks. These functions managed to identify the edges of defects and reproduce their geometry accurately. The specific nature of defects produced different responses in the virtual illumination functions, which depend on the relation between the thermal diffusivity of the base material and the diffusivity of the defect, so the response enable to identify the nature of the flaws. Defect depth identification was also provided by the virtual functions so the pulsed heating stimulation provided the best performance.

Two metrics have been used in the study to quantify the quality of detections provided by the VIFs: the SNR and the sharpness. The results obtained by the SNR demonstrate that the VIFs do not provide improvement in detection. On the other hand, the sharpness enable to identify key points in the time history related to the optimum detections, showing a high performance for defect depth characterization.

The next actions will include establishing relationships between the analysed parameters and the values of the VIFs and deducing recurrence laws to estimate values. Other VIFs for different processing algorithms will be analysed with theoretical data, and subsequently experimental tests will be carried out at the laboratory with calibrated samples to validate the results obtained in the theoretical study. Finally, an automatic classification system based on a convolutional neuronal network will be designed and trained with the experimental data obtained from the VIFs. 


\section{REFERENCES}

[1] Diakides M, Bronzino J.D, Peterson D.R. Medical infrared imaging: principles and practices. CRC press. 2012.

[2] López A, Molina-Aiz F, Valera D, Peña A. Determining the emissivity of the leaves of nine horticultural crops by means of infrared thermography. Scientia Horticulturae. 2012;137:49-58.

[3] Schaefer AL, Cook NJ, Church JS, Basarab J, Perry B, Miller C, Tong AKW. The use of infrared thermography as an early indicator of bovine respiratory disease complex in calves. Research in Veterinary Science. 2007;83(3):376-384.

[4] Shepard S.M, Lhota J.R, Rubadeux B.A, Wang D, Ahmed T. Reconstruction and enhancement of active thermographic image sequences. Optical Engineering. 2003;42(5);1337-1342.

[5] Ibarra-Castanedo C, Maldague X. Quantitative Infrared Thermography Journal. 2004;1(1):47-70.

[6] Marinetti S, Grinzato E, Bison P.G, Bozzi E, Chimenti M, Pieri G, Salvetti O. Statistical analysis of IR thermographic sequences by PCA. Infrared Physics \&Technology. 2004;46(1):85-91.

[7] Venegas P, Perán J, Usamentiaga R, Sáez de Ocáriz I. Projected thermal diffusivity analysis for thermographic nondestructive inspections. International Journal of Thermal Sciences. 2018;124:251-262. 